\title{
CATSPER2, a human autosomal nonsyndromic male infertility gene
}

\author{
Nili Avidan ${ }^{1}$, Hannah Tamary*,2,3,4, Orly Dgany ${ }^{2,3}$, Daniel Cattan ${ }^{6}$, \\ Alexandre Pariente ${ }^{7}$, Michel Thulliez ${ }^{8}$, Nicolas Borot ${ }^{9}$, Lucien Moati $^{10}$, \\ Alain Barthelme ${ }^{11}$, Lea Shalmon ${ }^{2,3}$, Tatyana Krasnov ${ }^{2,3}$, Edna Ben-Asher ${ }^{1}$, Tsvyia Olender ${ }^{1}$, \\ Miriam Khen $^{1}$, Issac Yaniv ${ }^{3,4}$, Rina Zaizov ${ }^{3,4}$, Hanna Shalev ${ }^{12}$, Jean Delaunay ${ }^{13}$, \\ Marc Fellous $^{14}$, Doron Lancet ${ }^{1}$ and Jacques S Beckmann ${ }^{1}$
}

\begin{abstract}
${ }^{1}$ Department of Molecular Genetics, The Crown Human Genome Center, The Weizmann Institute of Science, Rehovot 76100, Israel; ${ }^{2}$ Pediatric Hematology Laboratory, Felsenstein Research Center, Rabin Medical Center, Beilinson Campus, Petah Tikva, Israel; ${ }^{3}$ Sackler School of Medicine, Tel Aviv University, Israel; ${ }^{4}$ Pediatric Hematology, Schneider Children's Medical Center of Israel, Petah Tikva, Israel; Sackler Faculty of Medicine, Tel Aviv University, Israel; ${ }^{6}$ Service d'Hépatogastroentérologie, Centre Hospitalier, F-94195 Villeneuve-Saint-Georges, France; ${ }^{7}$ Unité

d'Hépatogastroentérologie, Centre Hospitalier, F-64046 Pau Cedex, France; ${ }^{8}$ Service d'Hématologie Biologique, Hôpital Henri Mondor, F 94010 Créteil Cedex, France; ${ }^{9}$ Centre d'Immunopathologie et de Génétique Humaine, CNRS, CHU Purpan F-31059 Toulouse, France; ${ }^{10}$ Service d' ORL, Hôpital Trousseau, Paris, France; ${ }^{11}$ Service d' ORL, Centre Hospitalier, Pau, France; ${ }^{12}$ Pediatric Department, Soroka Medical Center, Beer-Sheba, Israel; ${ }^{13}$ Service d'Hématologie, Hôpital de Bicêtre, Le Kremlin-Bicêtre, France; ${ }^{14}$ Institut Pasteur, Inserm 0021, 28 Rue du Dr Roux, 75015 Paris,
\end{abstract} France

In the course of positional cloning of the Congenital Dyserythropoietic Anemia type I (CDAI) [MIM 224120] gene on 15q15.1-15.3, we examined a family of French origin, in which the propositus suffered from asthenoteratozoospermia and nonsyndromic deafness in addition to CDAI. Two of his brothers had a similar phenotype. All three siblings were homozygous carriers of the CDA1 mutation as well as of a distally located $\sim 70 \mathrm{~kb}$ deletion of the proximal copy of a $106 \mathrm{~kb}$ tandem repeat on chromosome $15 q 15$. These repeats encode four genes whose distal copies may be considered pseudogenes. Lack of functional stereocilin and CATSPER2 (a voltage-gate cation channel expressed specifically in spermatozoa) may explain the observed deafness and male infertility phenotypes. To the best of our knowledge, the involvement of CATSPER2 in asthenoteratozoospermia is the first description of a human autosomal gene defect associated with nonsyndromic male infertility.

European Journal of Human Genetics (2003) 11, 497-502. doi:10.1038/sj.ejhg.5200991

Keywords: CATSPER2; Male-infertility; Tandem duplication; Contiguous gene syndromes; 15q15; Genomic deletion

\footnotetext{
*Correspondence: Dr H Tamary, Pediatric Hematology Oncology, Schneider's Children Medical Center of Israel, Petah Tikva 49202, Israel. Tel.: +972 3 9253669; fax: +97239253042 ;

E-mail: htamary@post.tau.ac.il

${ }^{15}$ Contributed equally to this work.

Received 26 July 2002; revised 4 February 2003; accepted 12 February 2003
}

Introduction

Several loci have been implicated in syndromic male infertility with oligospermia or azoospermia, such as the receptor genes for the follicle-stimulating hormone ${ }^{1,2}$ and $\mathrm{X}$-linked androgen. ${ }^{3-5}$ Despite an estimated prevalence of $\sim 0.3 \%$, only few genes or loci are known to be involved in human asthenospermia or asthenoteratozoospermia. These include the ' $9+0$ ' syndrome, in which the central pair of 
sperm cell microtubules is missing, leading to complete sperm immotility, ${ }^{6}$ and primary ciliary dyskinesia (PCD; MIM 242650), a group of heterogeneous disorders of unknown origin, usually inherited as autosomal recessive traits. The PCD phenotype is characterized by axonemal abnormalities of respiratory cilia and sperm tails, leading to bronchiectasis, sinusitis, sometimes associated with situs invertus (Kartagener syndrome), and male infertility with immobile spermatozoa. ${ }^{7}$ DNAH11, encoding for axonemal heavy-chain dynein type 11, was the first PCD gene identified. ${ }^{8,9}$ It was recently demonstrated that DNAH11 mutations account for situs inversus totalis but probably only for a minority of PCD cases. No gene involved in pure asthenospermia or asthenoteratozoospermia has been identified so far, although associations with mtDNA haplogroups ${ }^{10}$ and an autosomal dominant gene ${ }^{11}$ were reported. Two genes were recently shown to be involved in mouse sperm motility, CATSPER and CATSPER2.12,13 The CATSPER2 human homolog was localized to chromosome $15 q$, but no mutation affecting male infertility has been described in humans so far.

In the course of positional cloning of the Congenital Dyserythropoietic Anemia type I (CDAI) gene [MIM 224120], ${ }^{14}$ which was previously localized to chromosome $15 q 15.1-15.3,{ }^{15}$ we examined a family of French origin, in which the clinical and hematological features of the propositus (II-2, Figure 1a) and of his two affected brothers were compatible with CDAI (Table 1). However, unlike other CDAI patients, the brothers suffered also from sensorineural deafness, and a severe reduction in the percentage of motile spermatozoa in their ejaculates (Table 1). As a result of the perfect cosegregation of these additional features with their CDAI status, these patients were meticulously examined for the possible involvement of chromosome 15 in their condition. ${ }^{15}$ Detailed molecular analysis of this family's DNA samples revealed a $\sim 70 \mathrm{~kb}$ deletion in chromosome $15 q 15$, removing part of the CATSPER2 gene, and suggesting the importance of this gene for sperm motility in humans.

\section{Materials and methods Patients}

The propositus (II-2, Figure 1), a 56-year-old male from a nonconsanguineous family, from a small country town in southwest France, was found to be deaf in infancy. Later he proved to be sterile, and at the age of 53, presenting with mild jaundice and high serum ferritin, he was diagnosed, based on bone marrow electron microscopy, as having CDAI. Similar phenotypic findings were noted in his two affected brothers (Figure 1, Table 1). A detailed otorhinolaryngologic investigation (including tympanometric studies, a CT-scan of the temporal bones, brain stem-evoked potentials, videonystagmography, rotary/vestibular and caloric tests) revealed a moderate symmetrical sensorineur-

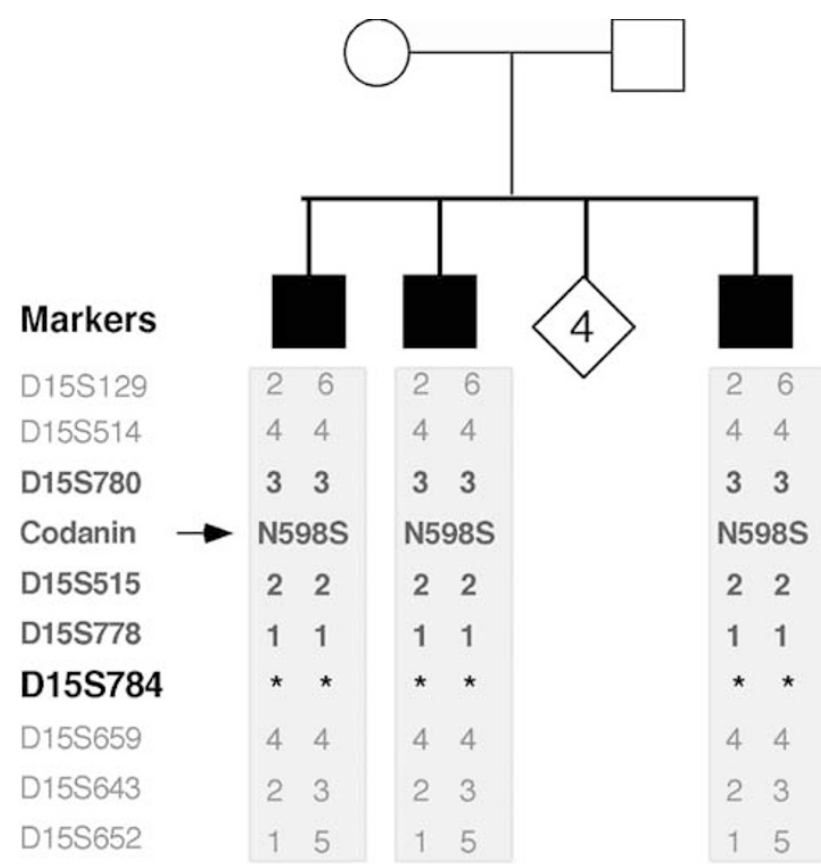

Figure 1 Pedigree of family with three affected male siblings suffering from CDAl, sensorineural deafness and lack of motile sperm cells, and their chromosome 15 q15 haplotypes. The location of the N598S codanin I mutation is indicated by an arrow. ${ }^{14}$ As D15S784 could not be amplified, it is marked in larger characters, while markers within the defined Bedouin CDAl linkage interval are in bold.

al hearing loss (40 dB) involving all frequencies (patients II2, II-4). Semen analysis showed a normal volume and sperm concentration. The motility and morphology of the spermatozoa were, however, abnormal, indicative of asthenoteratozoospermia. The percentage of motile cells at 1 and $2 \mathrm{~h}$ postejaculation was 3 and $1 \%$, respectively. The majority (91\%) of the sperm cells had short, coiled flagella. A similar clinical phenotype was observed in the third brother, although no semen analysis or audiographic tests were performed.

\section{Molecular analysis}

The genomic clones (AC011330, AC019011 and AC018512) used to validate the duplication were obtained from Multimegabase Sequencing Center, University of Washington, USA. Genotyping was performed according to standard procedures. Small copy-specific islands (mostly Alu-inserts) allowing discrimination between the proximal and distal copies of the repeat were identified by sequence alignments. To delineate the extent of the deletion, PCR reactions were performed with repeat-unit-specific primers as shown in Figure 2c. Sequence analysis of the CATSPER2 gene and pseudogene were performed by in silico alignment, sequence variants being subsequently validated and mapped on short- or long-range PCR fragments generated 
Table 1 Main clinical and laboratory features of the three affected brothers

\begin{tabular}{|c|c|c|c|}
\hline Patient & $11-1$ & $11-2$ & $11-4$ \\
\hline Age (years) & 58 & 56 & 54 \\
\hline Hemoglobin $(g / d l)$ & 13.9 & 12.3 & 13.1 \\
\hline MCV fL (Normal value $80-90 \mathrm{fl}$ ) & 99 & 98 & 97 \\
\hline Peripheral blood smear & $\begin{array}{l}\text { Basophilic stippling } \\
\text { and schiziocytes }\end{array}$ & $\begin{array}{l}\text { Basophilic stippling } \\
\text { and schiziocytes }\end{array}$ & $\begin{array}{l}\text { Basophilic stippling } \\
\text { and schiziocytes }\end{array}$ \\
\hline $\begin{array}{l}\text { Serum ferritin }(\mathrm{ng} / \mathrm{ml}) \\
\text { (normal value } 25-250 \mathrm{ng} / \mathrm{mL}) \\
\text { Bone marrow aspiration }\end{array}$ & 1550 & 1399 & 2100 \\
\hline $\begin{array}{l}\text { Bone marrow aspiration } \\
\text { Light microscopy }\end{array}$ & Intercellular chromatin bridges & Intercellular chromatin bridges & Intercellular chromatin bridges \\
\hline Electron microscopy & ND & $\begin{array}{l}\text { Spongy heterochromatin } \\
\text { Enlarged nuclear pores }\end{array}$ & ND \\
\hline Deafness & + & + & + \\
\hline Auditory testing & ND & Sensorineural deafness & Sensorineural deafness \\
\hline Infertility & + & + & \\
\hline Spermogram & ND & Asthenoteratospermia & Asthenoteratospermia \\
\hline
\end{tabular}

ND, not done, MCV, mean corpuscular volume.

on DNA from control individuals. Primer pairs were designed using Oligo primer software. All PCR reactions were performed according to standard procedures, and the products were subsequently sequenced using Big-Dyes dye terminators kit on an ABI 3700 sequencer (Perkin-Elmer/ Applied biosystems). Sequence comparisons were performed using a STADEN package ${ }^{16}$ and Sequencer software (gene codes cooperation: www.genecodes.com).

Full-length KIAA0377 transcripts were amplified, using long-range PCR of human cDNA libraries (Clontech) and of mRNAs from erythroid precursors grown in a two-phase liquid culture ${ }^{17}$ as previously described. ${ }^{18}$ The PCR products were cloned into a pCR2.1-TOPO vector to separate and sequence alternatively spliced variants expressed in the same tissue.

\section{Web-based databases and sequence analysis}

Genomic browsers: Santa Cruz, http://genome.ucsc.edu/ goldenPath/hgTracks.html;

Celera Human Genome Database (CHGD), http://cds.celera.com/;

Online Mendelian Inheritance in Man (OMIM), http:// www.ncbi.nlm.nih.gov/Omim.

Homology searches. KIAA0377 orthologs (H. sapiens KIAA0377: XP_012386, M. Musculus KIAA0377: AF50258, $H$. sapiens KIAA0433: NP_056031, D. melanogaster: AAF50947, C. elegans: T25770, A. thaliana: T51452, S. pombe: T41050, and $S$. cerevisiae: NP_013514) were identified upon a PSI-BLAST search of KIAA0377 against the NCBI nonredundant database. ${ }^{19}$ The KIAA0377-translated protein was analyzed by the following computer programs: EXPASY Proteomic tools containing a Profile scan and PROSITE software ${ }^{20}$ (http://www.expasy.ch/), BLOCKS ${ }^{21}$ (http://bioinformatics.weizmann.ac.il/blocks/) and YPDThe Yeast Proteome Database - (http://www.incyte.com/
sequence/proteome/databases/YPD.shtml) for KIAA0377 yeast ortholog function.

\section{Results}

Although no consanguinity was reported, the affected brothers were homozygous for the same N598S CDAI mutation $^{14}$ as well as for a battery of chromosome $15 \mathrm{q}$ markers (Figure 1a), which is suggestive of an unsuspected consanguineous antecedent. Marker D15S784 failed, however, to be amplified with several sets of primers, suggesting the existence of a possible deletion in these patients, which could account for their unique syndromic phenotype.

\section{Analysis of control chromosome and identification of an $\sim 106 \mathrm{~kb}$ tandem duplication}

A detailed analysis of control chromosomes led to the identification, distal to the location of the CDAI linkage interval, of an unsuspected $\sim 106 \mathrm{~kb}$ tandem duplication, involving four genes (Figure 2a): (a) The anonymous gene KIAA0377, encoding a 1408 aminoacid-long predicted protein of unidentified function; (b) creatine mitochondrial kinase 1 (CKMT1), which is crucial for energy metabolism, particularly in tissues with high-energy requirements; (c) a transcript expressed almost exclusively in the inner ear, encoding stereocilin (SRTC) $^{22}$ and (d) CATSPER2, a sperm-specific voltage-gated cation channel. ${ }^{13}$ Detailed in silico analysis revealed that although each pair of coding regions shows $>98.0 \%$ identity, three of the distal genes are likely to be pseudogenes (Figure 2a). The distal copy of KIAA0377 contains only the first 24 of 31 exons, that of SRTC has a stop codon in exon, ${ }^{22}$ and that of CATSPER2 differs at numerous positions in the transcript, including 
a AC018512

AC011330

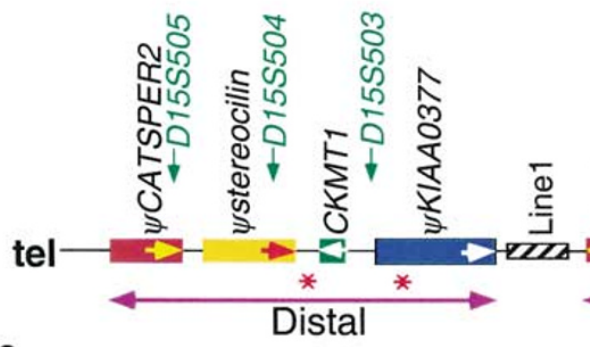

AC019011

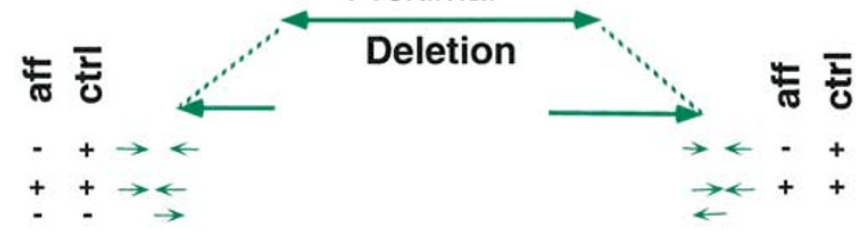

b

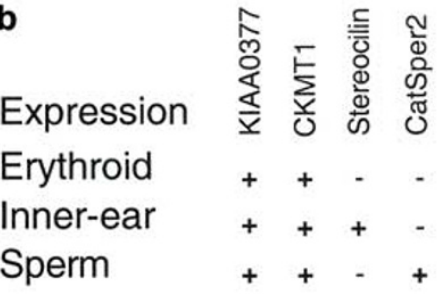

d

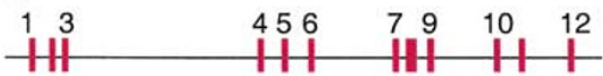

III

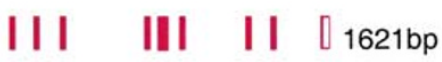

III

I I | II | $\left.\right|_{\text {6bp inexon splice variant }} ^{\text {I }}$

III

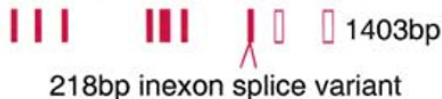

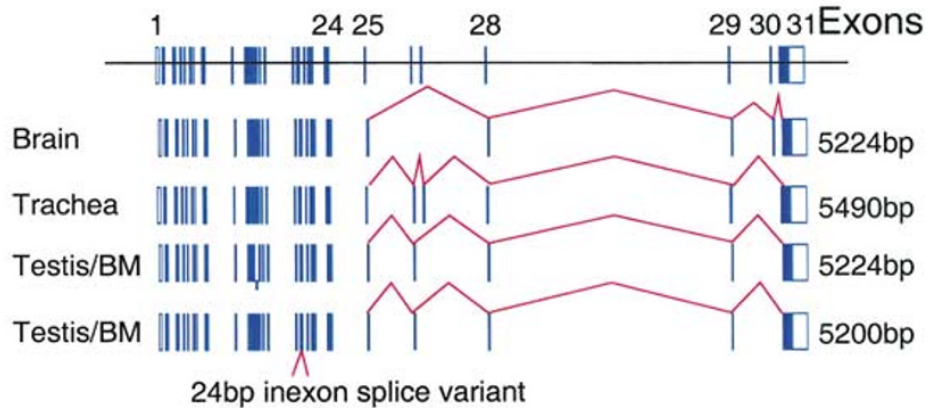

Figure 2 Expanded view of a duplicated genomic region on $15 q 15$ (telomere - left, centromere - right). (a) The blue, green and red lines represent different genomic clones (and accession numbers) according to which the duplication was identified and validated experimentally. Microsatellite marker positions are indicated by arrows on the genomic map. Duplicated markers are in green, unique markers in blue and D15S784 in red. Genes are shown as colored boxes with arrows indicating gene polarity. The unique line repeat separating the tandem duplications is indicated by a striped box. Pseudogenes are marked by a $\psi$ symbol. The purple arrow points to the proximal and distal duplication elements. (b) Expression patterns of KIAA0377 and CKMT1 (based on experimental and in silico data) and of stereocilin and CATSPER2 (based on in silico data). (c) Primers used to define the deletion boundaries are shown as sets of green arrows. The upper pair amplified only DNA from control individuals; the pair on the second line yielded the expected PCR fragments from both controls and affected brothers; the last set of primers, designed to span the deletion, failed to yield the bridging fragment. (d) Genomic structure of the proximal and distal CATSPER2 (red) and KIAA0377 (blue) genes. Coding and noncoding exons are depicted as filled and open boxes. Alternatively spliced transcripts of CATSPER2 (AF411817, AF411818, and AF411819 ${ }^{13}$ ) and KIAA0377 (AF502586, AF502587, AF502588, AF502589) are indicated, with their corresponding lengths to their right. Five single nucleotide polymorphisms (SNPs) were found in KIAA0377, including the two that cause amino-acid substitutions: a G356A (exon 3) and A4022G transition (exon 31) resulting, respectively, in an Arg77Gln and Glu1305Arg substitution.

base substitutions, gaps and a 1 bp deletion, creating a premature translation termination. CKMT1 is the only gene for which neither the distal nor the proximal copy shows any disruption of coding capacity (the distal copy differs in its cDNA by only two synonymous base substitutions: G1233A and C1263T). The D15S784 marker, which fails to be amplified in these patients, is located within a unique microregion separating the proximal SRTC and CATSPER2 loci. In addition, about $10 \mathrm{~kb}$ of a unique sequence containing a $6 \mathrm{~kb}$ line repeat (L1) separates the large duplicated segments (Figure 2a).
The affected patients carry a $\sim 70 \mathrm{~kb}$ deletion of the proximal copy of the repeat

In comparison with genomic DNA from healthy individuals which yielded both the proximal and distal copies of the Alu inserts, genomic DNA from the affected sibs yielded amplimers only of the Alu inserts present in the distal copy, thereby confirming the presence of a substantial deletion (Figure 2c). Figure 2c also shows the distal and proximal borders of the genomic deletion map, respectively, $1 \mathrm{~kb}$ centromeric to D15S505 and $2 \mathrm{~kb}$ centromeric to exon 24 of the KIAA0377 gene. None of the 
tested primer sets allowed amplification of the region bridging the deletion.

The affected siblings proved, based on local sequence divergence, to be homozygous carriers of a $\sim 70 \mathrm{~kb}$ deletion of the proximal copy of the repeat unit, encompassing D15S784 and the copy-specific Alu inserts, as well as the first 24 exons of KIAA0377, the entire coding sequences of both CKMT1 and SRTC, and the last two exons of CATSPER2, removing $225 \mathrm{bp}$ of the corresponding mRNA. D15S505, which is within the 10th intron of CATSPER2 and maps 5123 bp distal to D15S784, is not deleted (Figure $2 \mathrm{a}$ and $\mathrm{c})$.

\section{The deletion and the observed phenotype}

To determine to what extent this deletion could account for the observed deafness and sterile phenotypes encountered in the patients, and which of the encoded genes might underlie these pathologies, we examined each of these genes.

A similarity search revealed several proteins highly homologous to the KIAA0377 translated product. Although they all contain a single histidine acid phosphatase motif (data not shown), none has a known function, except for the yeast ortholog Asp1/Vip1, which is involved in the movement of actin patches. ${ }^{23}$ Next we, investigated the expression pattern of KIAA0377. RT-PCR amplification showed that it is expressed ubiquitously, yielding a complex pattern of alternatively spliced variants, the fulllength mRNAs being between 5400 and $5200 \mathrm{bp}$ long (Figure 2d). Hence, neither KIAA0377 nor the ubiquitous CKMT1 - involved in energy transduction in tissues with large, fluctuating energy demands, of which one intact copy is still present - appear a priori to be candidates for the etiology of the observed phenotypes. The lack of their proximal copies seems to have no visible phenotypic effect.

As for the remaining two genes, STRC and CATSPER2, the specificity of their expression pattern is compatible with the hypothesis that the observed deafness and infertility phenotypes are because of their respective inactivation (Figure 2b). Immunohistological labeling previously demonstrated that SRTC is expressed only in the inner ear, with intense staining along the hair bundle. ${ }^{22}$ Stereocilin is associated with stereocilia, the stiff microvilli forming the mechanoreception structure of sound stimulation. Furthermore, gene inactivating mutations in two DFNB16-linked families suggest that loss of stereocilin is involved in their deafness. ${ }^{22}$ Hence, the three brothers, having also lost the intact copy of the SRTC gene, belong to the same clinical and genetic deafness entity. CATSPER2 mRNA, however, is expressed during the meiotic stages of spermatogenesis, and all three of the cloned human testis cDNAs are transcribed from the proximal CATSPER2 gene, ${ }^{13}$ which is truncated in these patients (Figure 2c). CATSPER2 is thus a strong candidate for the observed infertility phenotype.

\section{Discussion}

Recent estimates suggest that as much as $5-10 \%$ of the human genome might be duplicated. These estimates of the number of duplicated segments suggest that rearrangements involving these sequences may play a prominent role in the etiology of a significant fraction of genetic disorders. ${ }^{24,25}$ Indeed, nonallelic homologous recombination (NAHR) between duplicated sequences may result in microdeletions, microduplications or inversions of genomic segments, causing Mendelian disorders, contiguous gene syndromes and whole-arm chromosome aberrations, depending on the size of the genomic segment involved. Of the 169 regions identified thus far as segmental duplications (and constituting roughly 1/10th of the genome $(298 \mathrm{Mb})), 24$ were found to be associated with genomic disorders. ${ }^{24}$ In the present study, we identified a $106 \mathrm{~kb}$ tandem duplication and a large genomic deletion that may underlie the observed deafness and male infertility phenotypes. Thus, these patients carry besides the CDAI mutation, a deletion inactivating four genes. CATSPER2, partially removed by the deletion, appears to be the best candidate for the etiology of the observed male infertility.

The CATSPER2 protein resembles the voltage-gated potassium channel. ${ }^{13}$ This ion channel is present on the sperm flagellum, suggesting its role in the regulation of sperm motility. CATSPER2 also strongly resembles another sperm-specific putative cation channel, namely CATSPER, ${ }^{12,13}$ whose targeted disruption results in male sterility in otherwise normal mice with reduced sperm motility and lack of cyclic AMP-induced $\mathrm{Ca}^{2+}$ influx. ${ }^{12}$ Both proteins possess a single, six-transmembrane-spanning repeat whose pore region and overall homology are close to a single domain of the much larger four-repeat Caselective ion channel. In both proteins, the pore-forming residue is located between the fifth and sixth transmembrane segments. The deletion found in our patients removes the last $225 \mathrm{bp}$ of CATSPER $2 \mathrm{mRNA}$. This fragment corresponds to the protein segment that mediates the actual channel pore complex ${ }^{13}$ suggesting that the natural pore complex of CATSPER2 is not formed in these patients, thereby affecting sperm motility, a functional assignment compatible with the observed phenotype, that is, asthenoteratozoospermia.

In view of their post-meiosis colocalization within the sperm flagellum, it has been suggested that these two proteins of the CATSPER family interact functionally and are part of a heterotetrameric Ca channel. ${ }^{13,26}$ Although coimmunoprecipitation failed to demonstrate any homoor heterotetramer formation, ${ }^{13}$ it would be of interest to measure the impact that the loss of one CATSPER family member has on the subcellular localization of the other. Finally, considering the decreased sperm motility and male infertility phenotype of the CATSPER mouse knockout model, these data warrant the systematic screening of 
CATSPER, which maps to 11q13.1, as an additional potential candidate in the generation of this particular phenotype. ${ }^{13}$ Both CATSPER and CATSPER2 may also be candidate genes for autosomal dominant asthenoteratozoospermia. $^{11}$

To sum up, the high structural and functional homology of CATSPER2 with its paralog, CATSPER, and the fact that inactivation of the latter leads to loss of sperm motility in mice, and the phenotype observed in our patients, indicate that disruption of CATSPER2 might underlie highly reduced sperm motility in man. Last but not least, to the best of our knowledge, the implication of CATSPER2 in asthenoteratozoospermia is the first description of an autosomal gene associated with nonsyndromic male infertility in humans.

\section{Acknowledgements}

We are indebted to Professor E Fibach from the Hematology Department, Hadassah Medical Center, Jerusalem, Israel, for culturing erythroid precursors from peripheral blood. This work was supported by a grant from the Israel Ministry of Health (HT), and by the J Maus and G Ceasemann-Maus, Chair in Pediatric Hematology (RZ). It is also supported by the Crown Human Genome Center, an Israel Ministry of Science grant to the National Laboratory for Genome Infrastructure, the Alfred Krupp Foundation, the German-Israeli Foundation for Scientific Research and Development, and The Weizmann Institute Glasberg, Levy, Nathan Brunschwig and Levine funds (DL). Doron Lancet holds the Ralph and Lois Silver Chair in Human Genomics. JSB is a recipient of the Hermann-Mayer chair.

\section{References}

1 Minegishi T, Nakamura K, Takakura $\mathrm{Y}$ et al: Cloning and sequencing of human FSH receptor cDNA. Biochem Biophys Res Commun 1991; 75: 1125-1130.

2 Aittomaki K, Lucena JL, Pakarinen P et al: Mutation in the folliclestimulating hormone receptor gene causes hereditary hypergonadotropic ovarian failure. Cell 1995; 82: 959-968.

3 Aiman J, Griffin JE: The frequency of androgen receptor deficiency in infertile men. J Clin Endocrinol Metab 1982; 54: $725-732$.

4 Gottlieb B, Trifiro M, Lumbroso $\mathrm{R}$ et al: The androgen receptor gene mutations database. Nucleic Acids Res 1997; 25: 158-162.

5 Tuerlings JH, Ligtenberg MJ, Kremer JA et al: Screening male intracytoplasmic sperm injection candidates for mutations of the follicle stimulating hormone receptor gene. Hum Reprod 1998; 3: 2098-2101.

6 Neugebauer DC, Neuwinger J, Jockenhovel F et al: E. '9+0' axoneme in spermatozoa and some nasal cilia of a patient with totally immotile spermatozoa associated with thickened sheath and short midpiece. Hum Reprod 1990; 5: 981-986.
7 Eliasson R, Mossberg B, Camner P et al: The immotile-cilia syndrome. A congenital ciliary abnormality as an etiologic factor in chronic airway infections and male sterility. N Engl J Med 1977; 297: 1-6.

8 Pennarun G, Escudier E, Chapelin C et al: Loss-of-function mutations in a human gene related to Chlamydomonas reinhardtii dynein IC78 result in primary ciliary dyskinesia. Am J Hum Genet 1999; 65: 1508-1519.

9 Bartoloni L, Blouin JL, Pan Y et al: Mutations in the DNAH11 (axonemal heavy chain dynein type 11) gene cause one form of situs inversus totalis and most likely primary ciliary dyskinesia. Proc Natl Acad Sci USA 2002; 99: 10282-10286.

10 Ruiz-Pesini E, Lapena AC, Diez-Sanchez C et al: Human mtDNA haplogroups associated with high or reduced spermatozoa motility. Am J Hum Genet 2000; 67: 682-696.

11 Tuerlings $\mathrm{JH}$, van Golde RJ, Oudakker AR et al: Familial oligoasthenoteratozoospermia: evidence of autosomal dominant inheritance with sex-limited expression. Fertil Steril 2002; 77: 415-418.

12 Ren D, Navarro B, Perez G et al: A sperm ion channel required for sperm motility and male fertility. Nature 2001; 413: 603-609.

13 Quill TA, Ren D, Clapham DE et al: A voltage-gated ion channel expressed specifically in spermatozoa. Proc Natl Acad Sci USA 2001; 98: 12527-31.

14 Dgany O, Avidan $\mathrm{N}$, Delaunay $\mathrm{J}$ et al: Congenital Dyserythropoietic Anemia Type I Is caused by mutations in codanin-1. Am J Hum Genet 2002; 71, 1467-1474.

15 Tamary H, Shalmon L, Shalev H et al: Localization of the gene for congenital dyserythropoietic anemia type I to a $<1$-cM interval on chromosome 15q15.1-15.3. Am J Hum Genet 1998; 62: 10621069 .

16 Bonfield JK, Smith K, Staden R: A new DNA sequence assembly program. Nucleic Acids Res 1995; 23: 4992-4999.

17 Pope SH, Fibach E, Sun J et al: Two-phase liquid culture system models normal human adult erythropoiesis at the molecular level. Eur J Haematol 2000; 64: 292-303.

18 Thongnoppakhun W, Wilairat P, Vareesangthip K et al: Long RTPCR amplification of the entire coding sequence of the polycystic kidney disease 1 (PKD1) gene. Biotechniques 1999; 26: 126-132.

19 Altschul SF, Gish W, Miller W et al: Basic local alignment search tool. J Mol Biol 1990; 215: 401-410.

20 Falquet L, Pagni M, Bucher P et al: The PROSITE database, its status in 2002. Nucleic Acids Res 2002; 30: 235-238.

21 Henikoff J, Greene E, Pietrokovski S et al: Increased coverage of protein families with the blocks database servers. Nucleic Acids Res 2000; 28: 228-230.

22 Verpy E, Masmoudi S, Zwaenepoel I et al: Mutations in a new gene encoding a protein of the hair bundle cause non-syndromic deafness at the DFNB16 locus. Nat Genet 2001; 29: 345-349.

23 Feoktistova A, McCollum D, Ohi R et al: Identification and characterization of Schizosaccharomyces pombe asp1(+), a gene that interacts with mutations in the Arp2/3 complex and actin. Genetics 1999; 152: 895-908.

24 Bailey JA, Gu Z, Clark RA et al: Recent segmental duplications in the human genome. Science 2002; 297: 1003-1007.

25 Stankiewicz P, Lupski JR: Genome architecture, rearrangements and genomic disorders. Trends Genet 2002; 18: 74-82.

26 Garbers DL: Ion channels. Swimming with sperm. Nature 2001; 579: 581-582. 